\title{
L'immunothérapie adoptive des tumeurs solides enfin réhabilitée
}

Francine Jotereau, Nathalie Labarrière, Nadine Gervois, Marie-Christine Pandolfino, Brigitte Dreno

$>$ Des modèles animaux de tumeurs expérimentales ont montré que le transfert (qualifié de transfert adoptif) de lymphocytes $T$ spécifiques d'antigènes de tumeurs pouvait induire une immunité passive, capable d'empêcher le développement de cellules cancéreuses greffées ou même d'éradiquer des métastases établies de ces cancers. Avant même l'identification d'antigènes de tumeurs humaines, le groupe de Steven Rosenberg aux États-Unis avait testé cette approche chez I'homme. Pour cela, il avait induit la prolifération des TIL (tumor infiltrating lymphocytes, lymphocytes $T$ qui infiltrent la tumeur) infiltrant des mélanomes malins, tumeurs souvent riches en lymphocytes $T$ spécifiques d'antigènes tumoraux, et les avait réinjectés au patient [1] (Figure 1). Pendant de nombreuses années, il fut cependant impossible non seulement de connaître le nombre exact de TIL spécifiques de tumeurs qui étaient réellement injectés dans ces essais, mais aussi d'apprécier leur capacité de migration dans la tumeur, puisque ces cellules étaient administrées par voie intraveineuse, donc à distance de la tumeur. La pertinence d'un tel traitement se fondait sur la seule évaluation clinique. Les difficultés techniques rencontrées par nombre de centres dans l'amplification des TIL, et surtout l'absence de résultats cliniques probants dans beaucoup de ces essais, avaient remis en cause le principe même de la thérapie adoptive pour le traitement des tumeurs solides. $0 r$, trois études récentes (dont une émanant à nouveau du groupe de S. Rosenberg [2]) ravivent l'intérêt pour la thérapie adoptive [2-5]. En effet, depuis les premiers essais, des méthodes de quantification des lymphocytes $T$ spécifiques d'antigènes ou d'épitopes ont été mises au point, et deux de ces études [2, 3] établissent que les lymphocytes T spécifiques d'antigènes de mélanome, sélectionnés et multipliés in vitro, puis réinjectés, peuvent survivre plusieurs semaines chez le patient et migrer dans la tumeur. Les trois études apportent des preuves cliniques de l'efficacité thérapeutique d'un tel transfert dans le traitement du mélanome malin.

Il a été établi, dans des modèles animaux, que la thérapie adoptive des tumeurs est plus efficace si elle est précédée d'un traitement immunosuppresseur [6]. Le groupe de S. Rosenberg a donc testé l'effet d'un tel conditionnement sur l'efficacité clinique des TIL, chez des patients atteints de mélanome métastatique. Une déplétion lymphocytaire sévère est obtenue par l'injection de cyclophosphamide puis de fludarabine. Les TIL spécifiques sont ensuite injectés par voie intraveineuse à ces patients, en association avec de fortes doses d'interleukine-2 (IL-2). Les résultats de cette étude sont rapportés dans la revue Science [2]. Ils montrent que les TIL injectés peuvent survivre plus de 4 mois, se multiplier et migrer dans la tumeur. Sur le plan clinique, une régression complète, partielle (diminution de la taille de toutes les tumeurs égale ou supérieure à $50 \%$ ) ou mixte
Inserm U.463,

Institut de Biologie/CHR,

9 , quai Moncousu,

44093 Nantes Cedex 1,

France.

jotereau@nantes.inserm.fr

(diminution de certaines tumeurs associée à la croissance ou à l'apparition d'au moins une autre) est obtenue chez 6 patients sur 13 . Cinq patients, dont trois chez lesquels la tumeur a régressé, ont développé une réponse autoimmune dirigée contre des cellules pigmentaires, induisant un vitiligo (zones cutanées dépigmentées) ou une uvéite. Une étude plus approfondie, réalisée chez deux de ces patients, montre que, malgré la polyclonalité des TIL injectés, c'est une population monoclonale de lymphocytes $\mathrm{T}^{\mathrm{C}} \mathrm{CD} 8^{+}$qui survit, et se multiplie dans le sang, jusqu'à représenter la majorité des lymphocytes T $\mathrm{CD}^{+}$circulants, pendant plusieurs mois. De plus, dans les deux cas, le clone survivant colonise les tumeurs et est spécifique du même antigène de mélanome MART-1/Melan-A, également exprimé par les cellules pigmentaires saines (présentes dans la peau et dans la rétine). Ces observations suggèrent que les réactions auto-immunes et les régressions tumorales, qui sont associées chez ces deux patients, résultent de l'activité directe de ces clones.

Deux patients ont par ailleurs développé des effets secondaires plus graves, un lymphome B-EBV (EpsteinBarr virus) et une infection par un virus syncytial, deux maladies dont l'émergence est favorisée par des traitements immunosuppresseurs.

Presque simultanément, le groupe de Greenberg publie les résultats d'un essai de thérapie adoptive de phase I mené chez 10 patients atteints de mélanome métastatique. Dans ce cas, 
des clones lymphocytaires T spécifiques soit de l'antigène MART-1/Melan-A, soit de l'antigène gp 100, ont été injectés, associés ou non à des injections sous-cutanées d'IL-2 aux patients qui n'avaient reçu aucun conditionnement immunosuppresseur préalable [3]. Les clones T ont été obtenus en cultivant à des concentrations cellulaires décroissantes (dilution limite) des lymphocytes du sang préalablement stimulés in vitro par l'antigène (Figure 1). Les résultats montrent que, 24 heures après l'injection, les clones injectés peuvent représenter jusqu'à $1,5 \%$ des lymphocytes $T$ CD $8^{+}$du sang et que leur demi-vie moyenne y est d'environ 17 ou 7 jours selon que le patient a ou non reçu de I'IL-2. Chez un patient pour lequel une biopsie tumorale a été effectuée trois jours après l'injection, les lymphocytes injectés représentaient $37,7 \%$ des TIL CD8 présents dans le fragment tumoral, mais seulement $0,8 \%$ de ces lymphocytes dans le sang, témoignant donc de leur accumulation et/ou de leur migration préférentielles dans la tumeur. Les réponses cliniques observées sont à première vue plus modestes que celles de l'étude de S. Rosenberg. Elles consistent essentiellement en une stabilisation de la maladie pouvant aller jusqu'à 21 mois. Les auteurs mentionnent de plus la disparition de l'antigène cible dans la tumeur de trois des cinq patients étudiés, suggérant que les clones injectés ont eu un effet cytotoxique direct sur les cellules tumorales exprimant l'antigène.

Ces deux études apportent donc pour la première fois des arguments forts justifiant la poursuite des essais de thérapie adoptive pour le traitement des tumeurs solides chez l'homme. En effet, elles démontrent que des lymphocytes $T$ $\mathrm{CD} 8^{+}$spécifiques d'un antigène tumoral, injectés en grand nombre (entre 10 à 100 milliards) avec de I'IL-2, peuvent survivre de façon prolongée chez le patient, coloniser différents sites, comme la tumeur et la peau, et y détruire les cellules qui expriment l'antigène cible. L'étude de $\mathrm{S}$. Rosenberg suggère de plus que si on fait précéder la thérapie adoptive d'une déplétion lymphocytaire sévère, et si on l'associe à de fortes doses d'IL-2, on peut accroître considérablement la survie de certains lymphocytes injectés. L'expansion préférentielle de clones reste cependant inexpliquée. Elle est aussi observée lors de mini-greffes de moelle osseuse allogénique, utilisées dans le traitement de leucémies après un conditionnement similaire. M. Dudley et al. [2] suggèrent aussi, au vu de résultats obtenus dans une étude antérieure [7], que les lymphocytes T CD4 ${ }^{+}$, présents parmi les TIL injectés, seraient indispensables à la survie des clones $\mathrm{CD}^{+}$. Les résultats de C. Yee et al. [3] infirment en partie cette hypothèse. $\varepsilon n$ effet, les clones T CD8 injectés survivent presque 1 mois dans leur essai. Les deux études suggèrent par ailleurs que les antigènes de différenciation mélanocytaire MART-1/Melan-A et gp100, bien qu'étant des antigènes $d u$ «soi normal » peuvent jouer le rôle d'antigènes tumoraux, cibles d'une immunité adoptive, alors que des immunothérapies, dont le principe repose sur l'injection de peptides de ces antigènes, n'avaient été accompagnées d'aucune réponse clinique.

Le risque de complications infectieuses ou cancéreuses que fait courir l'immunodépression associée à l'immunothérapie, dans l'étude de M. Dudley et al. [2], reste un problème sérieux, et rend indispensables l'évaluation de son utilité et l'analyse de son mécanisme. Des études chez l'animal suggèrent que la déplétion lymphocytaire contribue à l'élimination de populations $T$ suppressives du sang. Si c'est le cas chez I'homme, on pourrait envisager la déplétion sélective de cette popu-

Figure 1. Méthodes d'obtention de lymphocytes T CD8 spécifiques d'antigènes de mélanome pour la réalisation d'immunothérapies adoptives. TIL: tumor infiltrating lymphocytes.

Stimulation des leucocytes par un peptide antigénique

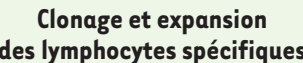


lation suppressive, ce qui éviterait d'exposer le patient au risque d'infections opportunistes. Il se peut cependant qu'une déplétion lymphocytaire totale soit essentielle à la survie des lymphocytes, du fait de mécanismes d'homéostasie.

La comparaison du bénéfice clinique obtenu dans ces deux études est difficile. $\varepsilon$ n effet, les résultats présentés par S. Rosenberg sont ceux d'une étude ouverte ne s'adressant qu'à des patients sélectionnés sur des critères précis, tandis que ceux rapportés par Greenberg sont ceux d'un même essai clinique. De plus, les régressions tumorales observées dans l'étude du groupe de S. Rosenberg concernent des localisations cutanées et ganglionnaires, connues comme étant les plus sensibles aux traitements, tandis que les stabilisations observées dans l'étude de Greenberg concernent des métastases viscérales plus résistantes.

Notre propre groupe vient de publier les résultats d'un essai d'immunothérapie adoptive par injection de TIL à des patients atteints de mélanome de stade III. II s'agit d'un essai randomisé dont le but est de comparer la survie sans rechute et la survie globale de patients ayant reçu comme traitement adjuvant des TIL et de I'IL-2 ou de I'IL-2 seule, injectée en sous-cutané, après exérèse complète de leur tumeur. Quatre-vingthuit patients ont été inclus dans cet essai. Bien qu'il n'y ait pas de différence significative dans la survie globale ni dans la survie sans rechute des patients ayant bénéficié de l'une ou l'autre stratégie, un bénéfice thérapeutique significatif est observé pour les patients traités par TIL, porteurs d'un seul ganglion envahi $(p<0,0285)$ [5]. Par ailleurs, nous avons montré, dans une étude réalisée a posteriori, que le bénéfice thérapeutique observé pour cette catégorie de patients était corrélé à la présence de lymphocytes T spécifiques de la tumeur autologue (sécrétant de I'IFN- $\gamma$ en réponse à la lignée tumorale) parmi les TIL injectés [4].
En résumé, ces travaux récents montrent que la thérapie adoptive, qui utilise le transfert de lymphocytes T autologues spécifiques d'antigènes de tumeur associé à de l'IL-2, permet la migration de ces lymphocytes dans les tumeurs et l'induction de réponses cliniques. La survie des lymphocytes, et peut-être l'efficacité clinique de cette approche, semblent pouvoir être augmentées par une déplétion lymphocytaire périphérique préalable, qui expose cependant le malade à des risques de maladies opportunistes. Des questions importantes restent à résoudre pour développer des protocoles plus sûrs et plus efficaces, applicables à d'autres pathologies cancéreuses que le mélanome. Ainsi, le rôle que pourraient jouer des lymphocytes $T$ $\mathrm{CD}^{+}$spécifiques d'antigènes de tumeur, et le rôle de la lymphodéplétion dans ces essais, doivent être précisés. $\bullet$

Adoptive immunotherapy in cancer: new hopes

\section{RÉFÉRENCES}

1. Rosenberg S, Packard B, Aebershold P, et al. Use of tumor-infiltrating lymphocytes and interleukin-2 in the immunotherapy of patients with metastatic melanoma. N Engl J Med 1988; 319 : 1676-80.

2. Dudley M, Wunderlich $P$, Robbins $P$, et al. Cancer regression and autoimmunity following clonal repopulation with anti-tumor lymphocytes and non-myeloablative conditioning. Science 2002; 298: 850-4.

3. Yee C, Thompson J, Byrd D, et al. Adoptive T cell therapy using antigen specific $C D 8^{+} \mathrm{T}$ cell clones for the treatment of patients with metastatic melanoma: in vivo persistence, migration and anti-tumor effect of transferred T cells. Proc Natl Acad Sci USA 2002; 99: 16168-73.
4. Labarrière N, Pandolfino MC, Gervois N, et al. Therapeutic efficacy of melanoma-reactive TIL injected in stage III melanoma patients. Cancer Immunol Immunother 2002; 51: 532-8.

5. Dreno B, Nguyen J, Khammari $A$, et al. Randomized trial of adoptive transfer of melanoma tumor infiltrating lymphocytes as adjuvant therapy for stage III melanoma. Cancer Immunol Immunother 2002; 51: 539-46.

6. North R.

Cyclophosphamidefacilitated adoptive immunotherapy of an established tumor depends on elimination of tumorinduced sppressor T cells J Exp Med 1982; 55 : 1063-74.

7. Dudley M, Wunderlich J, Yang J, et al. A phase I study of nonmyeloablative chemotherapy and adoptive transfer of autologous tumor antigen-specific $T$ lymphocytes in patients with metastatic melanoma. $J$ Immunother 2002; 25 : 243-51. 